\title{
Metal and 2D Material Interaction Investigated via HAADF STEM
}

Eileen Courtney ${ }^{1 *}$, Eoghan O’Connell ${ }^{1}$, Michelle Conroy ${ }^{1}$, Rahul Nair $^{2}$ and Ursel Bangert ${ }^{1}$

1. TEMUL, Department of Physics, School of Natural Sciences \& Bernal Institute, University of Limerick, Limerick, Ireland.

2. National Graphene Institute, School of Chemical Engineering and Analytical Science, University of Manchester, England.

*Corresponding author: Eileen.courtney@ul.ie

Since the discovery of graphene in 2004[1], there has been a surge of interest in two-dimensional (2D) materials research mainly due to the vast potential nano-electronic applications.[2,3] Their unusual physical phases result in their exotic electronic and opto-electronic properties.[4] More specifically 2D transition metal dichalcogenides (TMDCs) have recently garnered interest when it comes to electronics research, due to their varied electronic states and direct bandgap.[5,6] The ability to tune the bandgap allows for novel opto-electronic device applications[7,8]. Simulation studies have shown that different TMDC species could be perfect materials for a plethora of applications, from gas sensors[9], transistors[10], and energy cells[11].

The next challenge in these applications being realised is finding an experimentally stable electrical contacting solution. DFT calculations and theoretical studies from multiple sources have suggested various metals that might form favourable contacts with TMDCs [12]. Researchers have used AFM and Raman spectroscopy to characterize metals when grown or transferred to thin films[13]. However, there is sparse experimental evidence of the detailed interaction, i.e., on the atomic level. It is essential to study this contact/2D interaction (Figure 1 (a)) at the atomic scale as the 2Ds are only a few atoms thick themselves. The unique dimensional issue of atomically thin $2 \mathrm{D}$ based devices makes it essential to characterise the contacts at the atomic scale to understand the need to understand the fundamental physics hindering contact optimisation.

However, unless we find a stable electrical contacting solution, use of these 2Ds by the electronics industry will be unattainable. DFT calculations and theoretical studies from multiple sources have suggested various metals that might form favourable contacts with TMDCs [12]. Researchers have used AFM and Raman spectroscopy to characterize metals when grown or transferred to thin films[13]. However, there is sparse experimental evidence of the detailed interaction, i.e., on the atomic level.

Metals, such as palladium, gold etc. were investigated on physically exfoliated samples of 2D transition metal dichalcogenides $\left(\mathrm{MoS}_{2}, \mathrm{WS}_{2}\right.$, and $\left.\mathrm{WSe}_{2}\right)$, where the metals were introduced via e-beam deposition. Sputtering of angstrom quantities of metals on the flakes allowed for interaction of the metal and TMDC to be investigated on the $\AA$ scale in an aberration-corrected transmission electron microscope (Figure 1 (b)). Low energy sputtering of the metal introduced the metals to the 2D materials without causing damage to the thin flakes. We compared this experimental data to simulated HAADF images, to confirm and better understand the metal-2D interaction and interfacing. Effect of annealing and heat on stability of the metal2D interaction was investigated (Figure 2 (a) and (b)) [14]. 
References:

[1] KS Novoselov et al., Science 306 (2004), p. 666.

[2] D Kepaptsoglou et al., ACS Nano (2015), 151008021108008. doi:10.1021/acsnano.5b05305

[3] CR Dean et al., Nat. Nanotechnol. 5 (2010), p. 722.

[4] QH Wang et al., Nat. Nanotechnol. 7 (2012), p. 699.

[5] KF Mak et al., Phys Rev Lett. 105 (2010), p. 136805.

[6] A Laturia et al., npj 2D Mater. Appl. 2 (2018), p. 6.

[7] U Bangert et al., Ultramicroscopy 176 (2017), p. 31.

[8] N Lu et al., (2013). doi:10.1039/c3nr06072a

[9] W Yang et al., Inorg. Chem. Front. 3 (2016), p. 433.

[10] B Radisavljevic et al., Nat. Nanotechnol. 6 (2011), p. 147.

[11] M Pumera et al., J. Mater. Chem. (2012), 17.4.1. doi:10.1109/IEDM.2012.6479060

[13] S Cho et al., Science 349 (2015), p. 625.

[14] This work is funded by the Irish Research Council Scholarship GOIPG/2016/ 51.

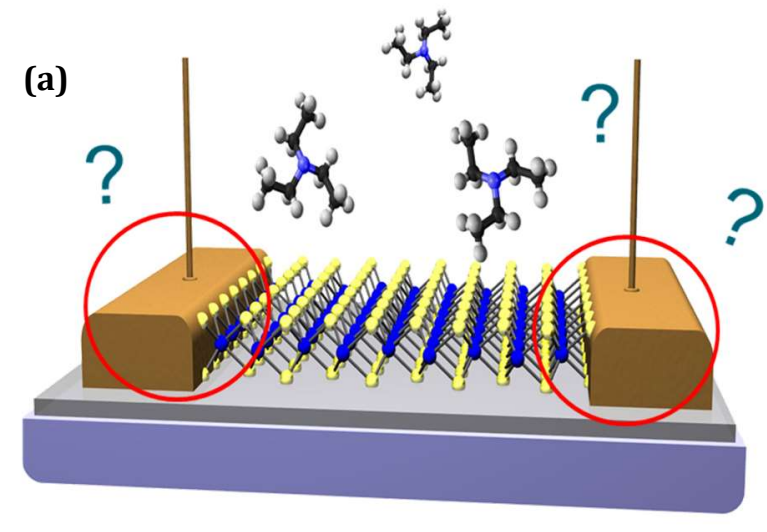

(b)

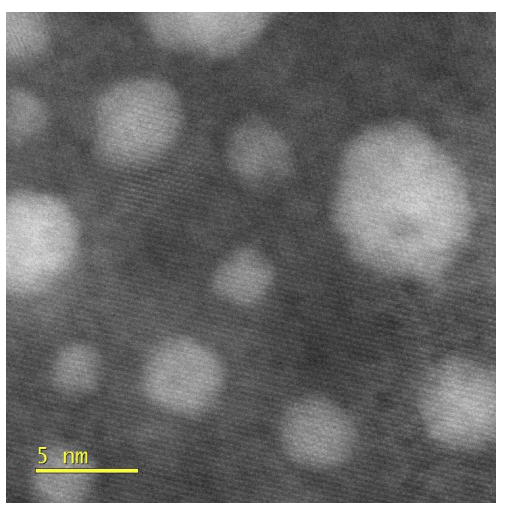

Figure 1. (a) Figure of MoS2 gas sensor as seen in [9]. The question remains what is happening at the metal 2D TMDC boundary. (b) STEM HAADF image taken on FEI TITAN of crystalline Pd nanoparticles on few-layer $\mathrm{WS}_{2}$.

(a)

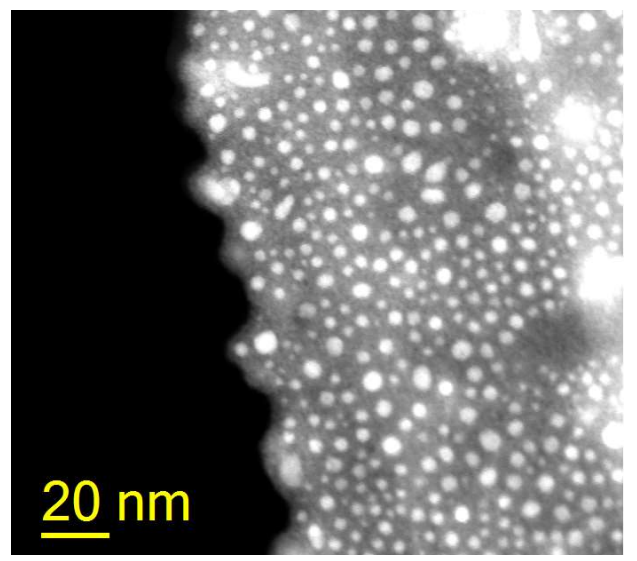

(b)

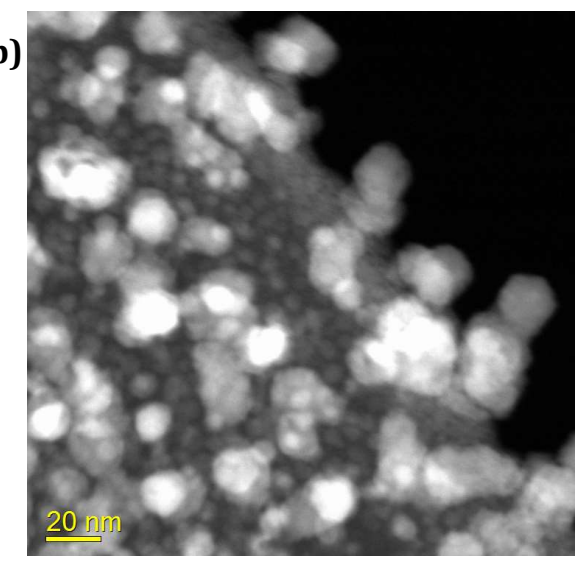

Figure 2. (a) $\mathrm{Pd}$ nanopartcles on monolayer $\mathrm{MoS}_{2}$ before annealing. (b) Pd nanopartcles on monolayer $\mathrm{MoS}_{2}$ after annealing at $200^{\circ} \mathrm{C}$, showing the evolution of the nanoparticles. 